\title{
ANALYTIC PERTURBATION OF THE TAYLOR SPECTRUM
}

\author{
ZBIGNIEW SLODKOWSKI
}

\begin{abstract}
Let $T_{1}(z), \ldots, T_{m}(z), z \in G \subset \mathbf{C}^{k}$, be analytic families of bounded operators in a complex Banach space $X$, such that for each $z \in G$ the operators $T_{i}(z)$ and $T_{j}(z), i, j=1, \ldots, n$, commute. MAIN RESULT: If $K(z)$ denotes the Taylor spectrum of the tuple $\left(T_{1}(z), \ldots, T_{m}(z)\right)$, then the set-valued function $K: G \rightarrow 2^{\mathrm{C}^{m}}$ is analytic. Analyticity of such set-valued functions is defined here by a simultaneous local maximum property of $k$-tuples of complex polynomials on the graph of $K$.
\end{abstract}

Introduction: Background and results. The following result, due to the author, is the starting point of this paper.

THEOREM. If $T(z), z \in G \subseteq \mathbf{C}$, is an analytic family of bounded operators on a Banach space, then the set-valued function $K(z)=\operatorname{sp}(T(z))$ is analytic in the following sense: the set

$$
U=\{(z, w): z \in G, w \notin K(z)\}
$$

is pseudoconvex.

The notion of analytic set-valued functions (shortly: analytic multifunction) referred to in the theorem was introduced by Oka [5]. The author applied it to spectral theory in $[\mathbf{8}, \mathbf{1 0}]$ (where two proofs of the theorem are given) and in [9] (where this theorem is generalized to families of unbounded operators); in [8] the notion of an analytic multifunction was examined in detail. The work of Aupetit $[\mathbf{1}]$ and Ransford [6] is related to this topic.

Since there is an almost-converse result to the above theorem (cf. [8, Theorem IV]) with $G$ bounded and the multifunction $K$ uniformly bounded, it seems that the analyticity condition (1) is suitable for characterizations of analytic perturbations of the spectrum.

The aim of this paper is to extend this approach to joint spectra. While there are many definitions of a joint spectrum for several commuting operators (cf. Slodkowski and Zelazko [15]), the one given by Taylor [16] seems to be the best one and is applied here. The Taylor spectrum is reviewed in $\S 1$. Now we recall the definition of analytic multifunctions in higher dimensions.

Received by the editors April 8, 1985. The contents of this paper have been presented to the American Mathematical Society on November 9, 1984, in San Diego, during the 815th meeting.

1980 Mathematics Subject Classification (1985 Revision). Primary 47A56, 47A60; Secondary $32 \mathrm{~F} 10$.

Key words and phrases. Analytic multifunction, local maximum property, joint spectrum, Taylor spectrum, exact complex, analytic perturbation. 
DEFINITION. A locally closed subset $X$ of $\mathbf{C}^{n}$ is said to have local maximum property of order $k$ (cf. $[\mathbf{1 1}, \mathbf{1 2}]$ ), where $0 \leq k \leq n-2$, if for every $(k+1)$ tuple of complex polynomials $p_{0}(z), \ldots, p_{k}(z)$ and for every compact subset $N$ of $X, \max y|N=\max y| \partial_{X} N$, where $y(z)=\min \left(\left|p_{1}(z)\right|, \ldots,\left|p_{k}(z)\right|\right)$ and $\partial_{X} N$ denotes the relative boundary of $N$ in $X$. An upper semicontinuous, compact valued multifunction $z \rightarrow K(z): G \rightarrow 2^{\mathrm{C}^{m}}$, where $G$ is open in $\mathbf{C}^{k}$, is said to be analytic (cf. [11]) if the set $\left\{(z, w) \in \mathbf{C}^{k+m}: z \in G, w \in K(z)\right\}$ has local maximum property of order $(k-1)$.

By $[8$, Theorem 3.2(vi)] this definition agrees with (1) if $k=m=1$; equivalent formulations of $k$-maximum property in terms of $q$-plurisubharmonic functions and $q$-pseudoconvex domains are given in [12]. We remark in passing that the notion of $k$-maximum property has already proved its usefulness in the context of uniform algebras, cf. [12].

THEOREM 1. Let $T_{1}(z), \ldots, T_{m}(z), z \in G \subseteq \mathbf{C}^{k}$, be analytic functions whose values are bounded linear operators acting on a Banach space. Assume that $T_{1}(z)$, $\ldots, T_{m}(z)$ commute for each $z \in G$ and set $K(z)=\operatorname{sp}\left(T_{1}(z), \ldots, T_{m}(z)\right)$, where $\mathrm{sp}(\cdot)$ denotes the Taylor spectrum. Then $z \rightarrow K(z): G \rightarrow 2^{\mathrm{C}^{m}}$ is an analytic multifunction.

This is the main result of this paper. Its proof depends on several other theorems and lemmas. We will outline now the general strategy of the proof and formulate those auxiliary results which are of independent interest.

Theorem 1 is easily reduced to the next two theorems. Spectrum $\sigma_{\pi, k}\left(T_{1}, \ldots, T_{n}\right)$ used in these theorems generalizes the approximate point spectrum and is a subset of the Taylor spectrum. Its definition is given in $\S 1$.

THEOREM 2. Let $(V, A)=\left(V_{1}, \ldots, V_{k} ; A_{1}, \ldots, A_{m}\right)$ be a commuting tuple of bounded operators on a complex Banach space. Then the set

$$
\operatorname{sp}(V, A) \backslash\left(\sigma_{\pi, k-1}(V) \times \mathbf{C}^{m}\right)
$$

is the graph of an analytic multifunction $K: G \rightarrow 2^{\mathrm{C}^{m}}$, where

$$
G=\operatorname{sp}(V) \backslash \sigma_{\pi, k-1}(V) .
$$

THEOREM 3. Let $P \subseteq \mathbf{C}^{k}$ be a bounded polydisc with nonempty interior and let $T_{1}(z), \ldots, T_{m}(z)$ be analytic functions defined in a neighborhood of $P$ whose values are bounded operators and commute for each $z \in P$. Then there exist a complex Banach space $Y$ and a tuple of commuting bounded operators $(V, A)=$ $\left(V_{1}, \ldots, V_{k} ; A_{1}, \ldots, A_{m}\right)$ acting on $Y$ such that

$$
\begin{gathered}
\operatorname{sp}(V)=\bar{P}, \quad \sigma_{\pi, k-1}(V)=\partial P \\
\operatorname{sp}(V, A)=\left\{(z, w): z \in P, w \in \operatorname{sp}\left(T_{1}(z), \ldots, T_{m}(z)\right)\right\} .
\end{gathered}
$$

The space $Y$ can be actually taken as the space $A_{+}(P, X)$ (if $T_{i}(z)$ acts on $X$ ) of all analytic $X$-valued functions on $P$ with absolutely convergent power series, which was studied in [14]. The proof of Theorem 3 is based on [14] and is given in $\S 5$, where also the proof of Theorem 1 is concluded.

The major part of the paper is devoted to the proof of Theorem 2 and related facts. The proof of Theorem 2 is reduced in $\S 2$ to Theorem 4 and Lemma 5 (to be 
given after the next definition) which deal with the Taylor spectrum of operators acting in quotient spaces.

DEFINITION. An upper semicontinuous multifunction $K: X \rightarrow 2^{Y}$ is said to be componentwise continuous if for every $x^{*} \in X$ and for every open subset $H$ of $Y$ such that $\partial H$ is disjoint from $K\left(x^{*}\right)$ and $H$ meets $K\left(x^{*}\right)$, there exists a neighborhood $V$ of $X^{*}$ such that $H$ intersects $K(x)$ for every $x \in V$.

If $T \in L(E, F)$ (= the space of all bounded operators from the Banach space $E$ to the Banach space $F$ ), denote as usual

$$
k(T)=\sup \{\operatorname{dist}(x, \operatorname{ker} T):\|T x\| \leq 1\} .
$$

THEOREM 4. Let $W$ be a compact topological space and let $Q: W \rightarrow L(E, F)$ and $T_{1}, \ldots, T_{m}: W \rightarrow L(F)$ be continuous. Assume that for each $\lambda \in W$ the range space of $Q(\lambda)$ is an invariant subspace for $T_{1}(\lambda), \ldots, T_{m}(\lambda)$. Then the set-valued function

$$
\lambda \rightarrow \operatorname{sp}\left(\tilde{T}_{1}(\lambda), \ldots, \tilde{T}_{m}(\lambda) ; F / \operatorname{Im} Q(\lambda)\right): W \rightarrow 2^{\mathbf{C}^{m}},
$$

(where $\tilde{T}_{i}(\lambda), 1 \leq i \leq m$, denotes the operator acting in $F / \operatorname{Im} Q(\lambda)$ induced by $\left.T_{i}(\lambda)\right)$ is upper semicontinuous and componentwise continuous.

LEMMA 5. Let $(V, A)=\left(V_{1}, \ldots, V_{k}, A_{1}, \ldots, A_{m}\right)$ be a commuting tuple of linear transformations acting in a complex linear space $X$. Assume that the Koszul complex of the tuple $V$, say $0 \rightarrow K^{0} \rightarrow K^{1} \rightarrow \cdots \rightarrow K^{k-1} \rightarrow K^{k} \rightarrow 0$ is exact at all places except for $K^{k}$. Then

$$
\operatorname{sp}\left(\tilde{A}_{1}, \ldots, \tilde{A}_{m} ; \tilde{X}\right)=\{w:(0, w) \in \operatorname{sp}(V, A)\},
$$

where $\tilde{A}_{i}$ denotes the operator induced by $A_{i}$ in the quotient space

$$
\tilde{X}=X /\left(\operatorname{Im} V_{1}+\cdots+\operatorname{Im} V_{k}\right), \quad 1 \leq i \leq m .
$$

The Koszul complex will be reviewed in $\S 1$. It suffices to say that assumptions of the lemma are fulfilled if $0 \notin \sigma_{\pi, k-1}\left(V_{1}, \ldots, V_{k}\right)$. Lemma 5 is proved in $\S 4$ by methods of homological algebra. $\S \S 2$ and 3 are devoted to the proof of Theorem 4 .

1. Local maximum property of a subset of the Taylor spectrum. In this section Theorem 2 is proved, under assumption that Theorem 4 and Lemma 5 hold. We recall, first, basic definitions related to the notion of the Taylor spectrum.

Let $X$ be a complex linear space and $T=\left(T_{1}, \ldots, T_{n}\right)$ be a commuting tuple of linear endomorphisms acting on $X$. Let $e=\left(e_{1}, \ldots, e_{n}\right)$ be an $n$-tuple of indeterminants and let $\Lambda=\bigwedge[e]$ denote the exterior algebra (over $\mathbf{C}$ ) with generators $e_{1}, \ldots, e_{n}$. Denote by $\bigwedge^{p}[e], 0 \leq p \leq n$, the subspace of $\Lambda[e]$ consisting of all elements of degree $p$, and set $\bigwedge^{p}[e, X]=X \otimes \bigwedge^{p}[e]$.

The Koszul complex of the tuple $T=\left(T_{1}, \ldots, T_{n}\right)$ is a cochain complex where $K^{p}=\bigwedge^{p}[e, X]$, and differentials $d^{i}$ are operators of "multiplication" by $T_{1} e_{1}+\cdots+$ $T_{n} e_{n}$. More specifically, if $x \in X$ and $i_{1}<i_{2}<\cdots<i_{p}$, then

$$
\begin{aligned}
d^{i}\left(x e_{i_{1}} \wedge e_{i_{2}} \wedge \cdots \wedge e_{i_{p}}\right)=\sum_{j=1}^{n} T_{j}(x) e_{j} \wedge e_{i_{1}} \wedge \cdots \wedge e_{i_{p}} \\
\quad=\sum_{s=1}^{p}(-1)^{s-1} \sum_{i_{s-1}<j<i_{s}} T_{j}(x) e_{i_{1}} \wedge \cdots \wedge e_{i_{s-1}} \wedge e_{j} \wedge e_{i_{s+1}} \wedge \cdots \wedge e_{i_{p}}
\end{aligned}
$$


if $x e_{i_{1}} \wedge \cdots \wedge e_{i_{p}}$ is identified with $x \otimes e_{i_{1}} \wedge \cdots \wedge e_{i_{p}}$.

The tuple $T$ is said to be regular, after Taylor $[\mathbf{1 6}, \mathbf{1 7}]$, if its Koszul complex (1.1) is exact. The Taylor spectrum of $T$ is defined as the set of all $z=\left(z_{1}, \ldots, z_{n}\right) \in \mathbf{C}^{n}$ such that the tuple $T-z I=\left(T_{1}-z_{1} I, \ldots, T_{n}-z_{n} I\right)$ is not regular, cf. Taylor [16]. Depending on the context, this spectrum will be denoted by $\operatorname{sp}(T), \operatorname{sp}(T ; X)$ or $\operatorname{sp}\left(T_{1}, \ldots, T_{n} ; X\right)$.

The subset $\sigma_{\pi, k}(T)$ of $\operatorname{sp}(T), 0 \leq k \leq n-1$, was defined by the author in [7]. Let $T$ be a commuting tuple of bounded operators in a complex Banach space $X$. A tuple $z \in \mathbf{C}^{n}$ does not belong to $\sigma_{\pi, k}(T)$ if the Koszul complex of the tuple $T-z I$ is exact at $K^{0}, K^{1}, \ldots, K^{k}$ and the differential $d^{k}$ has closed range. In the case of bounded operators on a Banach space the spectra $\operatorname{sp}(T)$ and $\sigma_{\pi, k}(T)$ are compact and nonempty. Other properties of these spectra will be recalled as needed in the proof. For further information on the Taylor spectrum and related functional calculus the reader is referred to $[\mathbf{1 6}, \mathbf{1 7}]$, and - for elementary proofs of some of its properties - to [7], where also the sets $\sigma_{\pi, k}(T)$ are studied.

The next lemma is a modification of [16, Lemma 2.1].

LEMMA 1.1. Let $X^{0}, X^{1}, \ldots, X^{n}$ be Banach spaces and let

$$
M_{n}=\left\{\left(d^{0}, d^{1}, \ldots, d^{n-1}\right): d^{i} \in L\left(X^{i}, X^{i+1}\right) \text { and } d^{i+1} \circ d^{i}=0,0 \leq i \leq n-1\right\} \text {, }
$$

and let $U_{n}$ be the set of all $\left(d^{0}, d^{1}, \ldots, d^{n-1}\right) \in M_{n}$ such that the complex

$$
0 \rightarrow X^{0} \stackrel{d^{0}}{\rightarrow} X^{1} \stackrel{d^{1}}{\rightarrow} \cdots \rightarrow X^{n-1} \stackrel{d^{n-1}}{\rightarrow} X^{n}
$$

is exact and $\operatorname{Im} d^{n-1}$ is closed. Then $U_{n}$ is open in $M_{n}$. Furthermore, the function $k\left(d^{n-1}\right)$ is continuous on $U$.

ProOF. Follows immediately from [14, Lemma 1.9] applied to each of the short sequences $X^{i-1} \stackrel{d^{i-1}}{\rightarrow} X^{i} \stackrel{d^{i}}{\rightarrow} X^{i+1}, 0 \leq i \leq n-1$. Q.E.D.

The following lemma strengthens the criterion for local maximum property of higher order given in [12, Theorem 5.1].

LEMMA 1.2. If $Z \subset \mathbf{C}^{n}$ is locally closed and does not have local maximum property of order $k, k \geq 0$, then there exist a point $z^{*} \in Z$, complex polynomials $p_{0}(z), \ldots, p_{k}(z)$, and positive numbers $\varepsilon>0, r>0$ such that

$$
\min _{i}\left|p_{i}(z)\right| \leq 1-\varepsilon\left|z-z^{*}\right|^{2}, \quad \text { for } z \in \mathbf{Z},\left|z-z^{*}\right|<r,
$$

while

$$
p_{0}\left(z^{*}\right)=p_{1}\left(z^{*}\right)=\cdots=p_{k}\left(z^{*}\right)=1 .
$$

PROOF. If $Z$ does not have local maximum property of order $k$, by [12, Theorem 5.1(iv)] there exist polynomials $p_{0}^{1}(z), \ldots, p_{k}^{1}(z)$, and a compact subset $N \subseteq Z$ such that $\max u|N>\max u| \partial_{Z} N$, where $u(z)=\log \min \left|p_{i}^{1}(z)\right|$, and $\partial_{Z} N$ denotes the topological boundary of $N$ relative to $Z$. By [12, Lemma 2.2] there exist a linear form $l(z)$, point $z^{*} \in Z$, and numbers $\varepsilon_{1}, r_{1}>0$ such that

$u\left(z^{*}\right)+\operatorname{Re} l\left(z^{*}\right)=0 \quad$ and $\quad u(z)+\operatorname{Re} l(z) \leq-\varepsilon_{1}\left|z-z^{*}\right|^{2}, \quad$ for $z \in Z,\left|z-z^{*}\right| \leq r$ or equivalently

$$
\begin{array}{r}
\left|\exp \left(u\left(z^{*}\right)+l\left(z^{*}\right)\right)\right|=1, \quad|\exp (u+l)(z)| \leq 1-\varepsilon_{2}\left|z-z^{*}\right|^{2}, \\
\text { for } z \in Z,\left|z-z^{*}\right|<r_{2},
\end{array}
$$


with different, positive $r_{2}, \varepsilon_{2}$. Observe that $|\exp (u+l)(z)|=\min _{i}\left|p_{i}^{1}(z) e^{l(z)}\right|$ and that the polynomial

$$
p_{i}^{\prime \prime}(z)=p_{i}^{1}(z)\left(1+l(z)-l\left(z^{*}\right)+\left(\frac{1}{2}\right)\left(l(z)-l\left(z^{*}\right)\right)^{2}\right) \exp \left(l\left(z^{*}\right)\right)
$$

differs from $p^{1}(z) e^{l(z)}$ only by terms of degree 3 or higher in $z_{j}-z_{j}^{*}, 0 \leq i \leq k, 1 \leq$ $j \leq n$. Therefore by $(1.5)$

$$
\min \left|p_{i}^{\prime \prime}\left(z^{*}\right)\right|=1, \quad \min \left|p_{i}^{\prime \prime}(z)\right| \leq 1-\varepsilon\left|z-z^{*}\right|^{2}, \quad \text { for }\left|z-z^{*}\right| \leq r, z \in Z,
$$

with some positive $\varepsilon$ and $r$. Choose $i(0)$ such that $\left|p_{i(0)}^{\prime \prime}\left(z^{*}\right)\right|=1$, and set $p_{i}(z)=$ $p_{i}^{\prime \prime}(z) \bar{p}_{i}^{\prime \prime}\left(z^{*}\right)$ if $\left|p_{i}^{\prime \prime}\left(z^{*}\right)\right|=1$ and $p_{i}(z)=p_{i(0)}(z)$ otherwise. The polynomials $p_{0}, p_{1}, \ldots, p_{k}$ thus defined satisfy (1.3) (with possibly smaller $r$ ) and, of course, $p_{i}\left(z^{*}\right)=1,0 \leq i \leq k$. Q.E.D.

We will prove now a lemma from which Theorem 2 follows easily. The next definition is inspired by Basener [2].

DEFINITION. Let $X$ be a compact subset of $\mathbf{C}^{n}$. The $k$ th rational hull of $X$, $k \geq 0$, is defined as the set of all $z^{*} \in \mathbf{C}^{n}$, such that for every $(k+1)$-tuple of complex polynomials, $p_{0}(z), \ldots, p_{k}(z)$, such that $p_{0}\left(z^{*}\right)=p_{1}\left(z^{*}\right)=\cdots=p_{k}\left(z^{*}\right)=0$, the variety $\left\{z \in \mathbf{C}^{n}: p_{i}(z)=0, i=0, \ldots, k\right\}$ has nonempty intersection with $X$. The $k$ th rational hull of $X$ will be denoted by $h_{k}^{r}(X)$. One observes that for $k=0$ the usual rational hull is obtained.

LEMMA 1.3. Let $T=\left(T_{1}, \ldots, T_{n}\right)$ be a commuting tuple of bounded operators on a Banach space $X$. Let $k \geq 0$ and $Z=\operatorname{sp}(T) \backslash h_{k}^{r}\left(\sigma_{\pi, k}(T)\right)$. Then $Z$ has local maximum property of order $k$.

ProOF. Suppose $Z$ does not have $k$-maximum property. By Lemma 1.2 there exist a point $z^{*}$, polynomials $p_{0}, \ldots, p_{k}$, and positive numbers $\varepsilon, r$ such that conditions (1.3), (1.4) hold. Since $z^{*} \notin h_{k}^{r}\left(\sigma_{\pi, k}(T)\right)$, there exist polynomials $w_{0}(z), \ldots, w_{k}(z)$ such that $w_{i}\left(z^{*}\right)=0,0 \leq i \leq k$, while $\left(w_{0}(z), \ldots, w_{k}(z)\right) \neq(0, \ldots, 0)$ for every $z \in \sigma_{\pi, k}(T)$. By this there exists a constant $a^{*}$ such that

$$
\left\|\left(p_{0}(z)-1, \ldots, p_{k}(z)-1\right)\right\|<a^{*}\left\|\left(w_{0}(z)^{3}, \ldots, w_{k}(z)^{3}\right)\right\|, \quad \text { for every } z \in \sigma_{\pi, k}(T) \text {. }
$$

Set $q_{i}(z)=p_{i}(z)+a^{*}\left(w_{i}(z)\right)^{3}, 0 \leq i \leq k$, and $M_{c}=\left\{z \in \mathbf{C}^{n}: q_{i}(z)=c, 0 \leq i \leq\right.$ $k\}$. Taking into account that $p_{i}\left(z^{*}\right)=1, w_{i}\left(z^{*}\right)=0$ and (1.6) we obtain that

$$
M_{c} \cap \sigma_{\pi, k}(T)=\varnothing \quad \text { for every } c \in[1-\delta, 1+\delta],
$$

for some positive $\delta$. Furthermore, applying the fact that $w_{i}(z)$, as polynomials in $z_{j}-z_{j}^{*}$, contain only terms of degree 3 or higher, and (1.3), we conclude that there exists a positive number $r$ such that

$$
M_{1} \cap B\left(z^{*}, r\right) \cap \sigma(T)=\left\{z^{*}\right\}, \quad \text { while } M_{c} \cap B\left(z^{*}, r\right) \cap \sigma(T)=\varnothing \text { for } c>1 .
$$

Define new operators $Q_{i}=q_{i}\left(T_{1}, \ldots, T_{n}\right), 0 \leq i \leq k$. By [7, remark after Lemma 2.7, p. 249], the spectrum $\sigma_{\pi, k}$ has the spectral mapping property and so

$$
\sigma_{\pi, k}(Q)=\left\{\left(q_{1}(z), \ldots, q_{k}(z)\right): z \in \sigma_{\pi, k}(T)\right\} .
$$

By this and (1.7) $(c, c, \ldots, c) \notin \sigma_{\pi, k}(Q)$ for $c \in[1-\delta, 1+\delta]$. Therefore if

$$
0 \rightarrow K^{0} \stackrel{d^{0}(c)}{\rightarrow} K^{1} \stackrel{d^{1}(c)}{\rightarrow} K^{2} \rightarrow \cdots \rightarrow K^{k \stackrel{d^{k}(c)}{\rightarrow}} K^{k+1} \rightarrow 0
$$


denotes the Koszul complex of $Q-c I=\left(Q_{1}-c I, \ldots, Q_{k}-c I\right)$, the complex is exact at $K^{0}, K^{1}, \ldots, K^{k}$ and $\operatorname{Im} d^{k}(c)$ is closed for $c \in[1-\delta, 1+\delta]$ (by the definition of $\left.\sigma_{\pi, k}\right)$. By Lemma 1.1

$$
\sup \left\{k\left(d^{k}(c)\right): 1-\delta \leq c \leq 1+\delta\right\}<+\infty,
$$

and so by Theorem 4 the set-valued function

$$
c \rightarrow \operatorname{sp}\left(\tilde{T}_{1}, \ldots, \tilde{T}_{n} ; K^{k+1} / \operatorname{Im} d^{k}(c)\right):[1-\delta, 1+\delta] \rightarrow 2^{\mathrm{C}^{n}}
$$

is componentwise continuous, where $\tilde{T}_{i}$ denote operators induced by $T_{i}, 1 \leq i \leq n$, on the quotient space $K^{k+1} / \operatorname{Im} d^{k}(c)=X /\left(\operatorname{Im}\left(Q_{0}-c I\right)+\cdots+\operatorname{Im}\left(Q_{k}-c I\right)\right)$. Observe now that it is enough to check the following assertion to complete the proof.

ASSERTION. $\operatorname{sp}\left(\tilde{T} ; K^{k+1} / \operatorname{Im} d^{k}(c)\right)=\sigma(T) \cap M_{c}$, for every $c \in \mathbf{C}$.

Indeed, if this is the case, and the set-valued function (1.9) is componentwise continuous, relations (1.8) cannot hold, which contradicts the initial assumption that $Z$ does not have $k$-maximum property.

To check the Assertion, we apply the spectral mapping theorem $[\mathbf{1 7}$, Theorem 4.8], (cf. also [15, Theorem 3.3] for an elementary proof) to the polynomial map $z \rightarrow\left(q_{0}(z)-c, \ldots, q_{k}(z)-c ; z\right): \mathbf{C}^{n} \rightarrow \mathbf{C}^{k+1+n}$ and obtain

$$
\begin{array}{r}
\operatorname{Sp}\left(Q_{0}-c I, Q_{1}-c I, \ldots, Q_{k}-c I, T_{1}, \ldots, T_{n} ; X\right) \\
=\left\{\left(q_{0}(z)-c, \ldots, q_{k}(z)-c, z\right): z \in \operatorname{sp}(T)\right\} .
\end{array}
$$

Using Lemma 5 and this identity we obtain that

$$
\begin{gathered}
\operatorname{sp}\left(\tilde{T}_{1}, \ldots, \tilde{T}_{n} ; X /\left(\operatorname{Im}\left(Q_{0}-c I\right)+\cdots+\operatorname{Im}\left(Q_{k}-c I\right)\right)\right) \\
=\left\{z \in \operatorname{sp}(T): q_{i}(z)-c=0,0 \leq i \leq k\right\}
\end{gathered}
$$

which proves the Assertion. Q.E.D.

ProOF OF THEOREM 2. Let $V, A, k$ be as in Theorem 2. By Lemma 1.3 the set $Z=\operatorname{sp}(V, A) \backslash h_{k-1}^{r}\left(\sigma_{\pi, k-1}(V, A)\right)$ has maximum property of order $k-1$. By $[\mathbf{7}$, Corollary 1.8] spectrum $\sigma_{\pi, k-1}$ has the projection property, and so

$$
\sigma_{\pi, k-1}(V, A) \subseteq \sigma_{\pi, k-1}(V) \times \mathbf{C}^{m} .
$$

Note that the latter set is equal to its $(k-1)$ th rational hull. Indeed, if $z^{*} \notin$ $\sigma_{\pi, k-1}(V), w^{*} \in \mathbf{C}^{m}$, one can define the polynomials $p_{i}(z, w)=z_{i}-z_{i}^{*}, 1 \leq i \leq k$. Then the variety $\left\{p_{i}(z, w)=0,1 \leq i \leq k\right\}$ contains $\left(z^{*}, w^{*}\right)$ but is disjoint from $\sigma_{\pi, k-1}$. This and (1.10) imply that the set $Z_{0}=\operatorname{sp}(V, A) \backslash\left(\sigma_{\pi, k-1}(V) \times \mathbf{C}^{m}\right)$ is contained in $Z$. Since $Z_{0}$ is a relatively open subset of $Z$, which has $(k-1)$-maximum property, $Z_{0}$ itself has $(k-1)$-maximum property by [12, Remark 2.4]. Q.E.D.

This completes the derivation of Theorem 2 from Theorem 4 and Lemma 5, whose proofs are given in next sections.

2. Continuous families of Banach spaces. For the proof of Theorem 4 on componentwise continuity of the Taylor spectrum we need several facts related to perturbations of exact complexes of Banach spaces. They are generally similar to those in $[\mathbf{1 6}, \S 2]$, but in our setting not only differentials but also spaces depend on a parameter. A distinctive feature of our treatment is the systematic use of continuous families of operators with closed ranges, say $T(\lambda)$, such that the function $k(T(\lambda))$ is locally uniformly bounded.

The proof of the next lemma can be extracted from [16, Proof of Lemma 2.1]. 
LEMMA 2.1. Let $X, Y$ be Banach spaces and $T \in L(X, Y)$, and $M, \delta$ be positive real numbers, $\delta<1$. Assume that for every $y \in Y$ with $\|y\|<1$ there exists $x \in X$ such that $\|x\| \leq M$ and $\|T x-y\| \leq \delta$. Then $T$ is onto and $k(T) \leq M /(1-\delta)$.

LEMMA 2.2. Let $X, Y, Z, F$ be Banach spaces and $A_{0} \in L(X, F), B_{0} \in L(Y, Z)$ and $T_{0} \in L(X, Y)$. Assume that $A_{0}$ and $B_{0}$ have closed ranges and $T_{0}\left(\operatorname{ker} A_{0}\right)=$ $\operatorname{ker} B_{0}$. Then for every $M>k\left(T_{0} \mid \operatorname{Im} A_{0}\right)$ and for every $C>0$ there exists $\varepsilon>$ 0 such that whenever $A \in L(X, F), B \in L(Y, Z)$ and $T \in L(X, Y)$ satisfy the conditions

$$
\begin{gathered}
\max \left(\left\|A-A_{0}\right\|,\left\|B-B_{0}\right\|,\left\|T-T_{0}\right\|\right)<\varepsilon, \\
k(A) \leq C \quad \text { and } \quad T(\operatorname{ker} A) \subseteq \operatorname{ker} B,
\end{gathered}
$$

then

$$
T(\operatorname{ker} A)=\operatorname{ker} B \quad \text { and } \quad k(T \mid \operatorname{ker} A) \leq M .
$$

PROOF. Assume that (2.1), (2.2) hold for some $\varepsilon>0$, which will be chosen later. We will show the assumptions of Lemma 2.1 hold for $T \mid \operatorname{ker} A: \operatorname{ker} A \rightarrow \operatorname{ker} B$ if $\varepsilon$ is small enough. Let $y \in \operatorname{ker} B,\|y\|<1$. Then $\left\|B_{0} y\right\|=\left\|\left(B_{0}-B\right) y\right\|<\varepsilon$ and so there exists $y^{\prime} \in Y$ such that $B_{0} y^{\prime}=B_{0} y$ and $\left\|y^{\prime}\right\|<k\left(B_{0}\right) \varepsilon$. Thus $\left\|y-y^{\prime}\right\|<1+k\left(B_{0}\right) \varepsilon$ and $y-y^{\prime} \in \operatorname{ker} B_{0}=T_{0}\left(\operatorname{ker} A_{0}\right)$. Therefore there exists $x_{0} \in \operatorname{ker} A_{0}$ such that $T x_{0}=y-y^{\prime}$ and

$$
\left\|x_{0}\right\|<K_{0}\left(1+k\left(B_{0}\right) \varepsilon\right), \quad \text { where } K_{0}=k\left(T_{0} \mid \operatorname{ker} A_{0}\right) .
$$

Since $\left\|A x_{0}\right\|=\left\|\left(A-A_{0}\right) x_{0}\right\|<\varepsilon K_{0}\left(1+k\left(B_{0}\right) \varepsilon\right)$, there exists $x^{\prime} \in X$ such that $A x^{\prime}=A x_{0}$ and

$$
\left\|x^{\prime}\right\|<C \varepsilon K_{0}\left(1+k\left(B_{0}\right) \varepsilon\right)
$$

(by (2.1)). Set $x=x_{0}-x^{\prime}$. Then $x \in \operatorname{ker} A$ and

$$
\|x\| \leq K_{0}(1+\varepsilon C)\left(1+\varepsilon K\left(B_{0}\right)\right) .
$$

Since $T x-y=\left(T-T_{0}\right) x_{0}-T x^{\prime}-y^{\prime}$, we get

$$
\begin{aligned}
\|T x-y\| & \leq \varepsilon\left\|x_{0}\right\|+\left(\left\|T_{0}\right\|+\varepsilon\right)\left\|x^{\prime}\right\|+\left\|y^{\prime}\right\| \\
& \leq \varepsilon\left(k\left(B_{0}\right)+K_{0}\left(1+k\left(B_{0}\right) \varepsilon\right)\left(1+\varepsilon C\left(\left\|T_{0}\right\|+\varepsilon\right)\right)\right) .
\end{aligned}
$$

If we denote the latter number by $\delta$, and $\varepsilon>0$ is small enough so that $\delta<1$, then by (2.5) and Lemma 2.1, $T(\operatorname{ker} A)=\operatorname{ker} B$ and $k(T) \leq k_{0}(1+\varepsilon C)\left(1+\varepsilon k\left(B_{0}\right)\right) /(1-\delta)$. Taking still smaller $\varepsilon>0$ we obtain $k(T)<M$. Q.E.D.

COROLLARY 2.3. Let $W$ be a topological space, $Y, Z, Y^{1}, Z^{1}$ be Banach spaces, and $A: W \rightarrow L(Y, Z), B: W \rightarrow L\left(Y^{1}, Z^{1}\right)$ and $T: W \rightarrow L\left(Y, Y^{1}\right)$ be continuous operator-valued functions. Assume that the functions $k(A(\lambda))$ and $k(B(\lambda))$ are locally uniformly bounded on $W$ and that $T(\lambda(\operatorname{ker} A(\lambda)) \subseteq \operatorname{ker} B(\lambda)$ for all $\lambda \in W$. Denote $W_{0}=\{\lambda \in W: T(\lambda)(\operatorname{ker} A(\lambda))=\operatorname{ker} B(\lambda)\}$. Then

(i) $W_{0}$ is open in $W$, $W_{0}$.

(ii) the function $k(T(\lambda) \mid \operatorname{ker} A(\lambda))$ is uniformly bounded on compact subsets of

This corollary follows immediately from Lemma 2.2. We need the following estimate for operators with closed ranges. 
Proposition 2.4. Let $X, Y, F$ be Banach spaces and $A \in L(X, F), T \in$ $L(X, Y)$. Define the operator $[A, T] \in L(X, A \times Y)$ by $[A, T] x=(A x, T x)$. Assume that $A$ has closed range. Then the operator $T \mid \operatorname{ker} A \in L(\operatorname{ker} A, Y)$ has closed range if and only if $[A, T]$ has closed range. Moreover

$$
k\left(T^{0}\right) \leq k([A, T]) \leq k\left(T_{0}\right)+k(T)+\|T\| k\left(T^{0}\right) k(T)
$$

where $T^{0}$ denotes $T \mid \operatorname{ker} A$ and $F \times Y$ has the maximum norm.

PROOF. Of course, it suffices to prove the inequalities (2.6).

Left-hand side inequality. Let $M>k([A, T])$. If $z \in \operatorname{Im} T^{0}$ and $\|z\|<1$, then $(0, z) \in \operatorname{Im}[A, T]$ and $\|(0, z)\|=\|z\|<1$. Therefore there exists $x \in X$ such that $[A, T] x=(0, z)$ and $\|x\|<M$. But this $x \in \operatorname{ker} A$ and so $T^{0}(x)=z$. We can conclude that $k\left(T^{0}\right)<M$, and so $k\left(T^{0}\right) \leq k([A, T])$.

Right-hand side inequality. Let $M>k(A), M^{0}>k\left(T^{0}\right)$ and $C>\|T\|$. Consider an arbitrary vector $(f, z) \in \operatorname{Im}[A, T]$ such that $\|(f, z)\|=\max (\|f\|,\|z\|)<1$. We have $(f, z)=(A x, T x)$ for some $x \in X$. Since $k(A)<M$, there exists $x^{\prime} \in X$ such that $A x^{\prime}=A x=f$ and $\left\|x^{\prime}\right\|<M\|f\|$. Therefore $x^{\prime}-x \in \operatorname{ker} A$, and since $k\left(T^{0}\right)<M^{0}$, there exists $x^{\prime \prime} \in \operatorname{ker} A$ such that $T\left(x^{\prime \prime}\right)=T\left(x^{\prime}-x\right)$ and so

$$
\left\|x^{\prime \prime}\right\|<M^{0}\left\|T\left(x^{\prime}-x\right)\right\| \leq M^{0}\left\|T x^{\prime}\right\|+M^{0}\|T x\| \leq M^{0} M C\|f\|+M^{0}\|z\| .
$$

Define $x_{0}=x^{\prime}-x^{\prime \prime}$. Since $A x_{0}=A\left(x^{\prime}-x\right)-A x^{\prime \prime}+A x=A x$ and $T x_{0}=$ $T\left(x^{\prime}-x\right)-T\left(x^{\prime \prime}\right)+T(x)=T(x)$, we have $[A, T] x_{0}=(f, z)$. Moreover

$$
\left\|x_{0}\right\| \leq\left\|x^{\prime}\right\|+\left\|x^{\prime \prime}\right\| \leq M\left(1+C M^{0}\right)\|f\|+M^{0}\|z\| .
$$

Therefore $k([A, T])<M^{0}+M+C M^{0} M$. Varying $M^{0}, M$ and $C$ suitably we obtain (2.6). Q.E.D.

The following lemma is an extension of [16, Theorem 2.1(b)]

LEMMA 2.5. Let $X, Y$ be Banach spaces, $W$ a compact topological space and $T: W \rightarrow L(X, Y)$ a continuous operator-valued function. Let $C(W, X)$ and $C(W, Y)$ denote the Banach spaces of all continuous $X$ - and $Y$-valued functions on $W$. Assume that all $T(\lambda), \lambda \in W$, have closed ranges, and $\sup _{\lambda \in W} k(T(\lambda))<\infty$. Then the operator $\tilde{T}: \mathcal{C}(W, X) \rightarrow \mathcal{C}(W, Y)$ defined by $(\tilde{T} f)(\lambda)=T(\lambda) f(\lambda)$ has closed range and $k(\tilde{T}) \leq \sup _{\lambda \in W} k(T(\lambda))$. Furthermore

$$
\operatorname{Im} \tilde{T}=\{g \in C(W, Y): g(\lambda) \in \operatorname{Im} T(\lambda), \text { for each } \lambda \in W\} .
$$

PROOF. We will apply Lemma 2.1 to the operator $\tilde{T}: \mathcal{C}(W, X) \rightarrow \tilde{Y}$, where

$$
\tilde{Y}=\{g \in \mathcal{C}(W, Y): g(\lambda) \in \operatorname{Im} T(\lambda), \lambda \in W\} .
$$

Let $g \in \tilde{Y},\|g\|<1$ (we consider sup norms in $C(W, X)$ and $C(W, Y)$ ). Fix $\delta>0$. Choose a finite covering $W_{1}, \ldots, W_{m}$ of $W$ and $\lambda_{i} \in W_{i}, 1 \leq i \leq m$, such that

$$
\left\|g(\lambda)-g\left(\lambda_{i}\right)\right\|<\delta \text { and }\left\|T(\lambda)-T\left(\lambda_{i}\right)\right\|<\delta \text { for } \lambda \in W_{i}, 1 \leq i \leq m \text {. }
$$

Fix $M>\sup _{\lambda \in W} k(T(\lambda))$. We can choose $x_{i}$ in $X$ such that $T\left(\lambda_{i}\right) x_{i}=g\left(\lambda_{i}\right)$ and $\left\|x_{i}\right\|<M, 1 \leq i \leq m$. Choose a nonnegative partition of unity, say $\left\{y_{i}(\lambda)\right\}_{i=1}^{m}$, subordinate to the covering $\left\{W_{i}\right\}$ and set $f(\lambda)=\sum_{i} \phi_{i}(\lambda) x_{i}, \lambda \in W$. Of course $\|f\|<M$ and

$$
\|g(\lambda)-T(\lambda) f(\lambda)\|=\left\|\sum_{i}\left(g(\lambda)-g\left(\lambda_{i}\right)\right) \phi_{i}(\lambda)\right\|<\delta, \quad \text { for all } \lambda \in W,
$$


(similarly as in [16, Proof of Theorem 2.1]), i.e. $\|g-\tilde{T} f\|<\delta$. If $\delta<1$, we obtain by Lemma 2.1 that $\tilde{T}$ is onto $\tilde{Y}$ and $k(\tilde{T}) \leq M /(1-\delta)$. Replacing $M$ and $\delta$ by suitable limit values one obtains $k(\tilde{T}) \leq \sup k(T(\lambda))$. Q.E.D.

In the proof of Theorem 4 we have to use Banach spaces of continuous functions whose values belong to subspaces depending on a parameter. The next two lemmas concern this situation.

LEMMA 2.6. Let $W$ be a compact topological space, $X$ and $F$ be Banach spaces and $A: W \rightarrow L(X, F)$ be a continuous operator-valued function such that $\sup _{\lambda \in W} k(A(\lambda))<\infty$. Then for every $\lambda_{0} \in W$ and for every $x_{0} \in \operatorname{ker} A\left(\lambda_{0}\right)$, there exists a continuous function $f \in C(W, X)$ such that $f\left(\lambda_{0}\right)=x_{0}$ and $A(\lambda) f(\lambda) \equiv 0$ on $W$.

Proof. Choose $M>\sup \{k(A(\lambda)): \lambda \in W\}$ and $\delta>0$. Denote $\tilde{X}=\{f \in$ $\mathcal{O}(W, X): A(\lambda) f(\lambda)=0, \lambda \in W\}$. We will apply Lemma 2.1 to the evaluation map $e \in L\left(\tilde{X}, \operatorname{ker} A\left(\lambda_{0}\right)\right)$ defined by $(e f)\left(\lambda_{0}\right)=f\left(\lambda_{0}\right)$. Consider $x_{0} \in \operatorname{ker} A\left(\lambda_{0}\right)$ such that $\left\|x_{0}\right\|<1$. Choose a neighborhood $W_{0}$ of $\lambda_{0}$ small enough so that $\left\|A(\lambda) x_{0}\right\|<\delta$ for $\lambda \in W_{0}$, and a continuous function $\phi$ on $W$ such that $0 \leq \phi \leq 1, \phi\left(A_{0}\right)=1$ and $\operatorname{supp} \phi \subseteq W_{0}$. Then $\sup \left\{\left\|A(\lambda)\left(\phi(\lambda) x_{0}\right)\right\|: \lambda \in W\right\}<\delta$. Since $\sup k(A(\lambda))<M$, by Lemma 2.5 there exists a function $f \in C(W, X)$ such that $A(\lambda) f(\lambda)=A(\lambda)\left(\phi(\lambda) x_{0}\right)$ for all $\lambda \in W$ and sup $\|f(\lambda)\|<M \delta$. Set $g(\lambda)=\phi(\lambda) x_{0}-f(\lambda)$. Then $g \in \tilde{X},\|g\| \leq$ $\left\|x_{0}\right\|+\delta M \leq 1+\delta M$ and $\left\|e(g)-x_{0}\right\|=\left\|f\left(\lambda_{0}\right)\right\|<M \delta$. If $\delta$ is chosen so that $\delta M<1$, Lemma 2.1 implies that $e$ is onto $\operatorname{ker} A\left(\lambda_{0}\right)$. Q.E.D.

LEMMA 2.7. Let $W$ be a compact topological space; $X, Y, F$ be Banach spaces and $A: W \rightarrow L(X, F)$ and $T: W \rightarrow L(X, Y)$ be continuous operator-valued functions. Let $\tilde{A} \in L(C(W, X), C(W, F))$ and $\tilde{T} \in L(C(W, X), C(W, Y))$ denote the operators $(\tilde{A} f)(\lambda)=A(\lambda) f(\lambda),(\tilde{T} f)(\lambda)=T(\lambda) f(\lambda), \lambda \in W$. Assume that

$$
\sup _{\lambda \in W} k(A(\lambda))<\infty \quad \text { and } \sup _{\lambda \in W} k(T(\lambda) \mid \operatorname{ker} A(\lambda))<\infty .
$$

Then $\tilde{T}(\operatorname{ker} \tilde{A})$ is a closed subspace of $C(W, Y)$. More specifically

$$
\tilde{T}(\operatorname{ker} \tilde{A})=\{g \in C(W, Y): g(\lambda) \in T(\lambda)(\operatorname{ker} A(\lambda)) \text { for all } \lambda \in W\} \text {. }
$$

Proof. Consider the operator $[\tilde{A}, \tilde{T}]: C(W, X) \rightarrow C(W, F) \times C(W, Y)$ defined by

$$
([\tilde{A}, \tilde{T}] f)(\lambda)=[A(\lambda), T(\lambda)] f(\lambda)=(A(\lambda) f(\lambda), T(\lambda) f(\lambda)) .
$$

By the uniform boundedness assumption (2.8) and inequality (2.6) of Proposition 2.4,

$$
\sup _{\lambda \in W} k([A(\lambda), T(\lambda)])<\infty,
$$

and so by Lemma $2.5,(2.7)[\tilde{A}, \tilde{T}]$ has closed range equal to

$$
\{(h, g) \in C(W, F) \times C(W, Y):(h(\lambda), g(\lambda)) \in \operatorname{Im}[A(\lambda), T(\lambda)] \text { for } \lambda \in W\} .
$$

We can now prove the equality (2.9). The inclusion $\subset$ is obvious. Concerning the reverse inclusion, take $g \in C(W, Y)$, such that $g(\lambda) \in T(\lambda)(\operatorname{ker} A(\lambda))$ for $\lambda \in W$. Then the pair $(0, g)$ belongs to the set $(2.10)$ and so is in the range of $[\tilde{A}, \tilde{T}]$, i.e., $(0, g(\lambda))=(A(\lambda) f(\lambda), T(\lambda) f(\lambda))$ for some $f$, that is $f \in \operatorname{ker} \tilde{A}$ and $g=\tilde{T} f$. Q.E.D.

The next fact is a direct consequence of Lemma 2.7. 
COROLlaRY 2.8. Let $W$ be a compact topological space, $E^{i}, F^{i}, 0 \leq i \leq m$, be Banach spaces and $Q^{i} \in C\left(W, L\left(E^{i}, F^{i}\right), 0 \leq i \leq m\right.$ and $d^{i} \in C\left(W, L\left(E^{i}, E^{i+1}\right)\right)$, $0 \leq i \leq m-1$, be continuous operator-valued functions. Assume that $k\left(Q^{i}(\lambda)\right), 0 \leq$ $i \leq m$, are uniformly bounded on $W$ and

$$
d^{i-1}(\lambda)\left(\operatorname{ker} Q^{i-1}(\lambda)\right)=\operatorname{ker} d^{i}(\lambda) \cap \operatorname{ker} Q^{i}(\lambda), \quad \lambda \in W, 0 \leq i \leq m .
$$

Denote by $\tilde{Q}_{i}$ the multiplication operator from $C\left(W, E^{i}\right)$ to $C\left(W, F^{i}\right)$ induced by $Q_{i}(\lambda), 0 \leq i \leq m$, and by $\delta^{i}: \operatorname{ker} Q^{i} \rightarrow \operatorname{ker} Q^{i+1}, 0 \leq i \leq m-1$, the operator $\left(\tilde{\delta}^{i} f\right)(\lambda)=d^{i}(\lambda) f(\lambda), f \in \operatorname{ker} \tilde{Q}^{i}$. Then the sequence

$$
0 \rightarrow \operatorname{ker} \tilde{Q}^{0} \stackrel{\tilde{\delta}^{0}}{\rightarrow} \operatorname{ker} \tilde{Q}^{1} \stackrel{\tilde{\delta}^{1}}{\rightarrow} \operatorname{ker} \tilde{Q}^{2} \rightarrow \cdots \stackrel{\tilde{\delta}^{m-1}}{\rightarrow} \operatorname{ker} \tilde{Q}^{m} \rightarrow 0
$$

is exact.

\section{Continuous perturbations of the Taylor spectrum.}

THEOREM 3.1. Let $E, F$ be complex Banach spaces; $W$ be a compact topological space and $Q: W \rightarrow L(E, F), T_{1}, \ldots, T_{m}: W \rightarrow L(F)$ be continuous operatorvalued functions. Assume that for each $\lambda \in W$ the subspace $\operatorname{ker} Q(\lambda)$ is invariant for $T_{1}(\lambda), \ldots, T_{m}(\lambda)$ and $\sup _{\lambda \in W} k(T(\lambda))<\infty$. Denote by $t_{i}(\lambda), 1 \leq i \leq$ $m, \lambda \in W$, the restricted operator $T_{i}(\lambda) \mid \operatorname{ker} Q(\lambda)$. Then the multifunction $\lambda \rightarrow$ $\operatorname{sp}\left(t_{1}(\lambda), \ldots, t_{m}(\lambda) ; \operatorname{ker} Q(\lambda)\right): W \rightarrow 2^{\mathbf{C}^{m}}$ is componentwise continuous.

We will prove this theorem in several steps and then obtain Theorem 4.

PROPOSITION 3.2. Under the assumptions of Theorem 3.1 the multifunction $\lambda \rightarrow \operatorname{sp}\left(t_{1}(\lambda), \ldots, t_{m}(\lambda) ; \operatorname{ker} Q(\lambda)\right): W \rightarrow 2^{\mathbf{C}^{m}}$ is upper semicontinuous.

ProOF. Denote $K(\lambda)=\operatorname{sp}\left(t_{1}(\lambda), \ldots, t_{m}(\lambda) ; \operatorname{ker} Q(\lambda)\right), \lambda \in W$. Of course, $K(\lambda) \subseteq P=\bar{D}(0, R) \times \cdots \times \bar{D}(0, R)$, if $R>\sup \left\{\left\|T_{i}(\lambda)\right\|: \lambda \in W, 1 \leq i \leq m\right\}$. For each $(\lambda, z) \in W \times P$ consider the Koszul complex

$$
0 \rightarrow \bigwedge^{0}[e, \operatorname{ker} Q(\lambda)] \rightarrow \bigwedge^{1}[e, \operatorname{ker} Q(\lambda)] \rightarrow \cdots \rightarrow \bigwedge^{m}[e, \operatorname{ker} Q(\lambda)] \rightarrow 0
$$

for the tuple $t_{1}(\lambda)-z_{1} I, \ldots, t_{m}(\lambda)-z_{m} I$; its differentials will be denoted by $\delta^{0}(\lambda, z), \delta^{1}(\lambda, z), \ldots, \delta^{m-1}(\lambda, z)$.

Denote by $G_{i}, 0 \leq i \leq m$, the set of all $(\lambda, z) \in W \times P$ such that the above complex is exact at places $\bigwedge^{i}[e, \operatorname{ker} Q(\lambda)]$ through $\bigwedge^{m}[e, \operatorname{ker} Q(\lambda)]$. We prove by induction on $i$ (going downwards) the following assertion.

ASSERTION. For every $0 \leq i \leq m$ the set $G_{i}$ is open and the functions $k\left(\delta^{i}(\lambda, z)\right), \ldots, k\left(\delta^{m}(\lambda, z)\right)$ are locally uniformly bounded on $G_{i}$.

Note that for $i=0$ this claim implies the proposition.

To prove the Assertion, denote by $\bigwedge^{i} Q(\lambda): \bigwedge^{i}[e, E] \rightarrow \bigwedge^{i}[e, F]$ the natural operator induced by $Q(\lambda)$. Then

$$
\begin{gathered}
\operatorname{ker} \bigwedge^{i} Q(\lambda)=\bigwedge^{i}[e, \operatorname{ker} Q(\lambda)] \\
G_{i}=\left\{(\lambda, z) \in G_{i+1}: \delta^{i-1}(\lambda, z)\left(\operatorname{ker} \bigwedge^{i-1} Q(\lambda)\right)=\operatorname{ker} \delta^{i}(\lambda, z)\right\} .
\end{gathered}
$$

Of course $k\left(\bigwedge^{i} Q(\lambda)\right)$ is uniformly bounded on $W \times P$. By these observations and Corollary $2.3 G_{m}$ is open in $W \times P$ and $k\left(\delta^{m-1}(\lambda, z)\right)$ is locally uniformly bounded on $G_{m}$. 
Assume now that the Assertion is true for $i+1, \ldots, m$; we will prove it for $i$. In order to be able to apply Corollary 2.3 , denote by $d^{i}(\lambda, z)$ the differential from $\bigwedge^{i}[e, F]$ to $\bigwedge^{i+1}[e, F]$ in the Koszul complex for the tuple $T_{1}(\lambda)-z_{1} I, \ldots, T_{m}(\lambda)-$ $z_{m} I$. Then the operator $\delta^{i}(\lambda, z): \operatorname{ker} \bigwedge^{i} Q(\lambda) \rightarrow \bigwedge^{i+1}[e, E]$ is equal to $d^{i}(\lambda, z)$ restricted to $\operatorname{ker} \bigwedge^{i}(Q(\lambda))$. Define

$$
B(\lambda, z)=\left[\bigwedge^{i} Q(\lambda), d^{i}(\lambda, z)\right]: \bigwedge^{i}[e, E] \rightarrow \bigwedge^{i}[e, F] \times \bigwedge^{i+1}[e, E],
$$

as in Proposition 2.4. Then $\operatorname{ker} B(\lambda, z)=\operatorname{ker} \delta^{i}(\lambda, z)$ and

$$
G_{i}=\left\{(\lambda, z) \in G_{i+1}: \delta^{i-1}(\lambda, z)\left(\operatorname{ker} \bigwedge^{i-1} Q(\lambda)\right)=\operatorname{ker} B(\lambda, z)\right\} .
$$

By the inductive assumption $k\left(d^{i}(\lambda, z) \mid \operatorname{ker} \bigwedge^{i} Q(\lambda)\right)$ is locally uniformly bounded on $G_{i+1}$, and so by Proposition $2.4 k(B(\lambda, z))$ is locally uniformly bounded on $G_{i+1}$. Now we are in the setting of Corollary 2.3 and by equation (3.4) the set $G_{i}$ is open and $k\left(\delta^{i-1}(\lambda, z)\right)$ is locally uniformly bounded on $G_{i}$. Q.E.D.

Similarly as in Lemma 2.7 consider now multiplication operators $\tilde{Q}: C(W, E) \rightarrow$ $C(W, F)$ and $\tilde{T}_{i}: C(W, E) \rightarrow C(W, E), 1 \leq i<m$, defined by $(\tilde{Q} f)(\lambda)=Q(\lambda) f(\lambda)$, $\left(\tilde{T}_{i} f\right)(\lambda)=T_{i}(\lambda) f(\lambda), \quad 1 \leq i \leq m$. Similarly, the operator-valued functions $t_{i}(\lambda), i=1, \ldots, m$, induce multiplication operators $\tilde{t}_{1}, \ldots, \tilde{t}_{m}$ acting in $\operatorname{ker} \tilde{Q}$. Of course, $\tilde{t}_{i}=\tilde{T}_{i} \mid \operatorname{ker} \tilde{Q}, 1 \leq i \leq m$.

LEMMA 3.3. Under the assumptions of Theorem 3.1

$$
\operatorname{sp}\left(\tilde{t}_{1}, \ldots, \tilde{t}_{m} ; \operatorname{ker} \tilde{Q}\right)=\bigcup\left\{\operatorname{sp}\left(t_{1}(\lambda), \ldots, t_{m}(\lambda) ; \operatorname{ker} Q(\lambda)\right): \lambda \in W\right\} .
$$

ProOF. Denote by $K$ the right side of equation (3.5). We prove first the inclusion $\operatorname{sp}\left(\tilde{t}_{1}, \ldots, \tilde{t}_{m} ; \operatorname{ker} \tilde{Q}\right) \subseteq K$. Fix an arbitrary $z^{*} \notin K$. We have to show that the Koszul complex

$$
0 \rightarrow \bigwedge^{0}[e, \operatorname{ker} \tilde{Q}] \rightarrow \bigwedge^{1}[e, \operatorname{ker} \tilde{Q}] \rightarrow \cdots \rightarrow \bigwedge^{m}[e, \operatorname{ker} \tilde{Q}] \rightarrow 0
$$

for the tuple $\tilde{t}_{1}-z_{1}^{*} I, \ldots, \tilde{t}_{m}-z_{m}^{*} I$ is exact. This complex can be identified, in a natural way, with the complex

$$
0 \rightarrow \operatorname{ker} \tilde{Q}^{0} \rightarrow \operatorname{ker} \tilde{Q}^{1} \rightarrow \cdots \rightarrow \operatorname{ker} \tilde{Q}^{m} \rightarrow 0
$$

where $\tilde{Q}^{i}$ denotes the multiplication operator from $C\left(W, \bigwedge^{i}[e, E]\right)$ to $C\left(W, \bigwedge^{i}[e, F]\right)$, $1 \leq i \leq m$, induced by the operator-valued function $\bigwedge^{i} Q(\lambda)$. Of course, the differentials in (3.7) are multiplication operators $\tilde{\delta}_{i}$ defined by $\left(\delta^{i} f\right)(\lambda)=\delta^{i}\left(\lambda, z^{*}\right) f(\lambda)$, $\lambda \in W, f \in \operatorname{ker} Q^{i}, 0 \leq i \leq m$, where $\delta^{i}\left(\lambda, z^{*}\right)$ are differentials of the complex (3.1). Since $z^{*} \notin K$ and by the Assertion in the proof of Proposition 3.2,

$$
\sup _{\lambda \in W} k\left(\delta^{i}\left(\lambda, z^{*}\right)\right)<\infty, \quad 0 \leq i \leq m-1
$$

and $\operatorname{ker} \delta^{i}\left(\lambda, z^{*}\right)=\operatorname{Im} \delta^{i-1}\left(\lambda, z^{*}\right)$ for every $\lambda \in W, 0 \leq i \leq m$, all the assumptions of Corollary 2.8 are fulfilled in this case. Therefore complex (3.7), and so also complex (3.6) are exact. Thus $z^{*} \notin \operatorname{sp}\left(\tilde{t}_{1}, \ldots, \tilde{t}_{m}\right)$, as required.

To show the reverse inclusion in (3.5) fix arbitrary $z^{*} \in K$; we will prove that $z^{*} \in \operatorname{sp}\left(\tilde{t}_{1}, \ldots, \tilde{t}_{m}\right)$. let $i \leq m$ be the largest index such that for some $\lambda \in W$ the complex

$$
0 \rightarrow \operatorname{ker} Q^{0}(\lambda) \rightarrow \operatorname{ker} Q^{1}(\lambda) \rightarrow \cdots \rightarrow \operatorname{ker} Q^{m}(\lambda) \rightarrow 0
$$


with differentials $\delta^{0}\left(\lambda, z^{*}\right), \ldots, \delta^{m-1}\left(\lambda^{*}, z^{*}\right)$ is not exact at $\operatorname{ker} Q^{i}(\lambda)$. Choose $\lambda^{*}$ and $y_{0} \in \operatorname{ker} Q^{i}\left(\lambda^{*}\right)$ such that $y_{0} \in \operatorname{ker} \delta^{i}\left(\lambda^{*}, z^{*}\right) \backslash \delta^{i-1}\left(\lambda^{*}, z^{*}\right)\left(\operatorname{ker} Q^{i-1}\left(\lambda^{*}\right)\right)$. By the choice of $i$ the set $W \times\left\{z^{*}\right\}$ is contained in $G_{i}$ (cf. (3.2)) and by the Assertion $\sup _{\lambda \in W} k\left(\delta^{i}\left(\lambda, z^{*}\right)\right)<\infty$. Therefore, if we define $B\left(\lambda, z^{*}\right)$ by (3.3), then by Proposition $2.4 \sup _{\lambda \in W} k\left(B\left(\lambda, z^{*}\right)\right)<\infty$. Since $\operatorname{ker} \delta^{i}\left(\lambda, z^{*}\right)=\operatorname{ker} B\left(\lambda, z^{*}\right)$, we obtain, by Lemma 2.6, that there exists a function $g \in \operatorname{ker} \tilde{\delta}_{i}$ such that $g\left(\lambda^{*}\right)=y_{0}$. It is clear that $g \notin \tilde{\delta}^{i-1}\left(\operatorname{ker} \tilde{Q}^{i-1}\right)$. Q.E.D.

COROLLARY 3.4. With the assumptions and notations of Theorem 3.1 let $h(z)$ be an analytic function defined in a neighborhood of the set

$$
\bigcup_{\lambda \in W} \operatorname{sp}\left(t_{1}(\lambda), \ldots, t_{m}(\lambda), \operatorname{ker} Q(\lambda)\right),
$$

and let $h(t(\lambda))=h\left(t_{1}(\lambda), \ldots, t_{m}(\lambda)\right) \in L(\operatorname{ker} Q(\lambda))$ be the operator obtained by the analytic functional calculus. Then the family of operators $\lambda \rightarrow h(t(\lambda))$ is continuous in the sense that for every $f \in \operatorname{ker} \tilde{Q}$, the function $\lambda \rightarrow h(t(\lambda)) f(\lambda): W \rightarrow E$ is continuous.

Proof. $h$ is also analytic in a neighborhood of $\operatorname{sp}\left(\tilde{t}_{1}, \ldots, \tilde{t}_{m}, \operatorname{ker} \tilde{Q}\right)$ by Lemma 3.3 , and so we may consider the operator $h(\tilde{t})=h\left(\tilde{t}_{1}, \ldots, \tilde{t}_{m}\right)$, defined by Taylor's analytic functional calculus, cf. [17]. Since $h(\tilde{t})$ maps $\operatorname{ker} \tilde{Q}$ into itself, it suffices to show that for every $\lambda \in W$ and for every $f \in \operatorname{ker} \tilde{Q}$

$$
h(t(\lambda)) f(\lambda)=(h(\tilde{t}) f)(\lambda) .
$$

Denote by $u: \operatorname{ker} \tilde{Q} \rightarrow \operatorname{ker} Q(\lambda)$ the evaluation operator $u f=f(\lambda)$. Then $u \tilde{t}_{i}=$ $t_{i}(\lambda) u, 1 \leq i \leq m$, i.e., $u$ is an intertwining operator for the tuples $\tilde{t}$ and $t(\lambda)$. Therefore by [17, Proposition 4.5] $u$ intertwines $h(t)$ and $h(t(\lambda))$, i.e., $u h(\tilde{t})=$ $h(t(\lambda)) u$, which is identical with (3.8). Q.E.D.

PROOF OF ThEOREMS 3.1 AND 4. Denote $K_{\lambda}=\operatorname{sp}\left(t_{1}(\lambda), \ldots, t_{k}(\lambda), \operatorname{ker} Q(\lambda)\right)$. To show that the multifunction $\lambda \rightarrow K_{\lambda}$ is componentwise continuous, consider an arbitrary $\lambda^{*} \in W$ and an open subset $H \subseteq \mathbf{C}^{m}$ such that $\partial H \cap K_{\lambda^{*}}=\varnothing$ and the set $K^{\prime}=H \cap K_{\lambda}$. is nonempty. By the upper semicontinuity of $K_{\lambda}$ we can choose a compact neighborhood $W^{*}$ of $\lambda^{*}$ such that $\partial H \cap K_{\lambda}=\varnothing$ for all $\lambda \in W^{*}$. Denote by $V$ the set of all $\lambda \in W^{*}$ such that the set $K_{\lambda}^{\prime}=H \cap K_{\lambda}$ is nonempty. To complete the proof of Theorem 3.1 we have to show that $V$ is a neighborhood of $\lambda^{*}$.

Let $h(z) \equiv 1$ on $H$ and 0 on $\mathbf{C}_{m} \backslash \bar{H}$. Clearly $h$ is analytic in a neighborhood of $K_{\lambda}$ for $\lambda \in W^{*}$. Let $P_{\lambda}=h\left(t_{1}(\lambda), \ldots, t_{m}(\lambda)\right) \in L(\operatorname{ker} Q(\lambda))$ be the operator produced by Taylor's functional calculus. By $\left[\mathbf{1 7}\right.$, Theorem 4.9] $P_{\lambda}$ is a projection such that $\operatorname{sp}\left(t_{1}(\lambda)\left|\operatorname{Im} P_{\lambda}, \ldots, t_{m}(\lambda)\right| \operatorname{Im} P_{\lambda}\right)=K_{\lambda}$. Since the Taylor spectrum is nonempty for operators acting in a space of positive dimension, cf. [17, §3, Corollary], $V=$ $\left\{\lambda \in W^{*}: P_{\lambda} \neq 0\right\}$. Since $\lambda \rightarrow P_{\lambda}$ is continuous in the sense of Corollary $3.4, V$ is a neighborhood of $\lambda^{*}$. More specifically, take $y \in \operatorname{Im} P_{\lambda^{*}},\|y\|=1$. By Lemma 2.6 there exists $f \in \operatorname{ker} \tilde{Q}$ such that $f\left(\lambda^{*}\right)=y$. By Corollary $3.4 g(\lambda)=P_{\lambda} f(\lambda)$ is a continuous function on $W^{*}$, and $g\left(\lambda^{*}\right)=y$. Thus $P_{\lambda} \neq 0$ in a neighborhood of $\Lambda^{*}$.

To obtain Theorem 4, we apply Theorem 3.1 to the dual operator families $Q(\lambda)^{*}, T_{1}(\lambda)^{*}, \ldots, T_{m}(\lambda)^{*}$. This works because $k\left(Q(\lambda)^{*}\right)=k(Q(\lambda))$ and

$$
\operatorname{sp}\left(\tilde{T}_{1}(\lambda), \ldots, \tilde{T}_{m}(\lambda) ; Y / \operatorname{Im} Q(\lambda)\right)=\operatorname{sp}\left(t_{1}(\lambda), \ldots, t_{m}(\lambda) ; \operatorname{ker} Q(\lambda)^{*}\right),
$$

where $t_{i}(\lambda)=T_{i}(\lambda)^{*} \mid \operatorname{ker} Q(\lambda)^{*} \in L\left(\operatorname{ker} Q(\lambda)^{*}\right)$ by [16, Theorem 3.6]. Q.E.D. 
4. Applications of homological algebra. The proof of Lemma 5 can be conveniently presented with the use of the notion of a double complex. We recall now, after Hilton and Stammbach [4, $\S \mathrm{V} .1]$ basic definitions related to double complexes.

A double complex $K$ is a system of modules $\left\{K^{p, q}: p, q=0, \pm 1, \pm 2, \ldots\right\}$, with two families of differentials $\delta^{\prime}: K^{p, q} \rightarrow K^{p+1, q}$ and $\delta^{\prime \prime}: K^{p, q} \rightarrow K^{p, q+1}$ satisfying the relations

$$
\delta^{\prime} \delta^{\prime}=0, \quad \delta^{\prime \prime} \delta^{\prime \prime}=0, \quad \delta^{\prime \prime} \delta^{\prime}+\delta^{\prime} \delta^{\prime \prime}=0 .
$$

A double complex is called bounded if all but a finite number of its modules are zero modules. There is a standard way to construct a cochain complex out of $K$, called totalization. The complex Tot $K$ consists of modules (Tot $K)^{n}, n=0, \pm 1, \pm 2, \ldots$ which are direct sums of modules $K^{p, n-p}, p=0, \pm 1, \pm 2, \ldots$, and differentials $\delta:=\delta^{\prime}+\delta^{\prime \prime}:(\operatorname{Tot} K)^{n} \rightarrow(\operatorname{Tot} K)^{n+1}$.

We need the following property of double complexes.

LEMMA 4.1. Let $K=\left\{K^{p, q}, \delta^{\prime}, \delta^{\prime \prime}\right\}$ be a bounded double complex such that $K^{p, q}$ is nonzero only when $0 \leq p \leq k$ and $0 \leq q \leq m$ ( $k$ and $m$ given). Assume that for every $p \neq k$ and for every $q$

$$
\operatorname{Im}\left\{\delta^{\prime}: K^{p-1, q} \rightarrow K^{p, q}\right\}=\operatorname{ker}\left\{\delta^{\prime}: K^{p, q} \rightarrow K^{p+1, q}\right\} .
$$

Then the complex $\operatorname{Tot}(K)$ is exact if and only if the quotient complex

$$
\left\{K^{k, q} / \delta^{\prime}\left(K^{k-1, q}\right): q=0, \pm 1, \pm 2, \ldots\right\},
$$

with differentials $\tilde{\delta}^{\prime \prime}$ induced by $\delta^{\prime \prime}$ is exact.

ProOF. Observe first that by (4.1) $\delta^{\prime \prime}\left(\delta^{\prime}\left(K^{k-1, q}\right)\right) \subseteq \delta^{\prime}\left(K^{k-1, q+1}\right)$, and so the differentials $\tilde{\delta}^{\prime \prime}$ are well defined. By the same argument, if we set $M^{p, q}$ equal to $\delta^{\prime}\left(K^{k-1, q}\right)$ if $p=k$, and to $K^{p, q}$ otherwise, $M=\left\{M^{p, q}\right\}$ becomes a double subcomplex of $K$ (with differentials $\delta^{\prime}$ and $\delta^{\prime \prime}$ suitably restricted). Consequently we can define the quotient double complex $L=K / M=\left\{K^{p, q} / M^{p, q}\right\}$ with naturally induced differentials. Then the short sequence of double complexes $0 \rightarrow M \rightarrow K \rightarrow$ $L \rightarrow 0$ is exact, by which we mean that at each bidegree the sequence of modules $0 \rightarrow M^{p, q} \rightarrow K^{p, q} \rightarrow L^{p, q} \rightarrow 0$ is exact. We need the following assertions whose simple proofs are omitted.

ASSERTION 1. An exact sequence of double complexes $0 \rightarrow M \rightarrow K \rightarrow L \rightarrow 0$ induces an exact sequence of totalizations

$$
0 \rightarrow \operatorname{Tot}(M) \rightarrow \operatorname{Tot}(K) \rightarrow \operatorname{Tot}(L) \rightarrow 0 .
$$

ASSERTION 2 (Khelemskii [3, Proposition 7]). If all rows or all columns of a bounded double complex are exact, then its totalization is an exact complex.

By (4.2) and the construction of $M$, the double complex $M$ has all rows exact (and is bounded) and by the last assertion $\operatorname{Tot}(M)$ is exact. Thus the terms $H^{n}(\operatorname{Tot}(M))$ in the long exact cohomology sequence of the short sequence (4.4) are all zero and so $\operatorname{Tot}(K)$ is exact if and only if $\operatorname{Tot}(L)$ is.

Observe finally that $L^{p, q}=0$, if $p \neq k$ and $L^{k, q}=K^{k, q} / \delta^{\prime}\left(K^{k-1, q}\right)$ and all $\delta^{\prime}$ induce zero differentials in $L$. Thus $(\operatorname{Tot}(L))^{n}=L^{k, n-k}$, with differentials in $\operatorname{Tot}(L)$ induced by $\delta^{\prime \prime}$ alone. Of course exactness of $\operatorname{Tot}(L)$ is equivalent to the exactness of the complex (4.3). Q.E.D. 
ProOF OF LemMa 5 . It is enough to prove that $0 \notin \mathrm{sp}(V, A ; X)$ if and only if $0 \notin \operatorname{sp}(\tilde{A} ; \tilde{X})$.

Considering the Koszul complex of the tuple $(V, A)=\left(V_{1}, \ldots, V_{k}, A_{1}, \ldots, A_{m}\right)$ it is convenient to divide the generators of the exterior algebra (cf. $\S 1$ ) into two subsets, say $e=\left(e_{1}, \ldots, e_{k}\right)$ and $f=\left(f_{1}, \ldots, f_{m}\right)$. The $n$th module of the Koszul complex of $(V, A)$ is now $\bigwedge^{n}[(e, f), X]$. This representation suggests the use of the following double complex.

$K^{p, q}, 0 \leq p \leq k, 0 \leq q \leq m$, is defined as the subspace of $\bigwedge^{p+q}[(e, f), X]$ spanned by elements

$x e_{i_{1}} \wedge \cdots \wedge e_{i_{p}} \wedge f_{j_{i}} \wedge \cdots \wedge f_{j_{q}}, \quad x \in X, 1 \leq i_{1}<\cdots<i_{p} \leq k, 1 \leq j_{1}<\cdots<j_{q} \leq m$.

That is $K^{p, q}$ is the direct sum of $\left(\begin{array}{l}k \\ p\end{array}\right)\left(\begin{array}{c}m \\ q\end{array}\right)$ copies of $X$. (For all the other pairs of indices $p, q$ space $K^{p, q}$ is set as zero.) We make the family $K=\left\{K^{p, q}: p, q=\right.$ $0, \pm 1, \pm 2, \ldots\}$ into a double complex by defining two families of differentials

$$
\begin{aligned}
& \begin{aligned}
& \delta^{\prime}\left(x e_{i_{1}} \wedge \cdots \wedge e_{i_{p}} \wedge f_{j_{1}}\right.\left.\wedge \cdots \wedge f_{j_{q}}\right) \\
&=\sum_{s=1}^{k}\left(V_{s} x\right) e_{s} \wedge e_{i_{1}} \wedge \cdots \wedge e_{i_{p}} \wedge e_{j_{1}} \wedge \cdots \wedge e_{j_{q}} \\
& \delta^{\prime \prime}\left(x e_{i_{1}} \wedge \cdots \wedge e_{i_{p}} \wedge f_{j_{1}} \wedge \cdots \wedge f_{j_{q}}\right) \\
&=(-1)^{p} \sum_{s=1}^{m}\left(A_{s} x\right) e_{i_{1}} \wedge \cdots \wedge e_{i_{p}} \wedge\left(f_{s} \wedge f_{j_{1}} \wedge \cdots \wedge f_{j_{m}}\right) .
\end{aligned}
\end{aligned}
$$

By the commutativity of $(V, A)$ and properties of exterior multiplication, $\delta^{\prime}$ and $\delta^{\prime \prime}$ satisfy the rules (4.1) and so $\left\{K, \delta^{\prime}, \delta^{\prime \prime}\right\}$ is a double complex. One can check by inspection that the totalization of this double complex is the Koszul complex of $(V, A)$.

Observe next that for every $0 \leq q \leq m$ the horizontal complex $\left\{K^{p, q}: p=\right.$ $\left.0, \pm 1, \pm 2, \ldots, \delta^{\prime}\right\}$ is a direct sum of $\left(\begin{array}{c}m \\ q\end{array}\right)$ copies of the Koszul complex

$$
0 \rightarrow K^{0} \rightarrow K^{1} \rightarrow \cdots \rightarrow K^{k} \rightarrow 0
$$

of the tuple $V$ acting on $X$. By the assumption of Lemma 5 , complex $0 \rightarrow K^{0} \rightarrow$ $K^{1} \rightarrow \cdots \rightarrow K^{k}$ is exact and so the same holds for the horizontal complex

$$
0 \rightarrow K^{0, q} \stackrel{\delta^{\prime}}{\rightarrow} K^{1, q} \stackrel{\delta^{\prime}}{\rightarrow} \cdots \stackrel{\delta^{\prime}}{\rightarrow} K^{k-1, q} \stackrel{\delta^{\prime}}{\rightarrow} K^{k, q}
$$

Thus all the assumptions of Lemma 5.1 are fulfilled and so $\operatorname{Tot}(K)$ is exact if and only if the complex (4.3) is exact. It is easy to observe, however, that the latter complex is canonically isomorphic to the Koszul complex of the tuple $\tilde{A}=\left(\tilde{A}_{1}, \ldots, \tilde{A}_{m}\right)$ acting on the quotient space $\tilde{X}=X /\left(\operatorname{Im} V_{1}+\cdots+\operatorname{Im} V_{k}\right)$. (Note that by (4.5) the usual differential $d^{n}: \bigwedge^{n}[f, \tilde{X}] \rightarrow \bigwedge^{n+1}[f, \tilde{X}]$ of this Koszul complex and that induced by $\delta^{\prime \prime}$ are $(-1)^{k}$ multiples of each other.) Q.E.D.

With this proof we completed all the steps needed to prove Theorem 2 . The next algebraic lemma will be needed in the derivation of Theorem 1 from Theorem 2. 
LEMMA 4.2. (a) Let $V=\left(V_{1}, \ldots, V_{k}\right)$ be a commuting tuple of endomorphisms on a linear space $X$. Let

$$
X_{p}=\operatorname{Im} V_{1}+\operatorname{Im} V_{2}+\cdots+\operatorname{Im} V_{p}, \quad \text { for } 0<p \leq k,
$$

and $X_{0}=(0)$. If $\tilde{V}_{p}$, the linear transformation induced by $V_{p}$ on $X / X_{p-1}$ has zero kernel for $1 \leq p \leq k$, then the sequence $0 \rightarrow K^{0} \rightarrow K^{1} \rightarrow \cdots \rightarrow K^{k-1} \rightarrow K^{k}$ is exact, where

$$
0 \rightarrow K^{0} \rightarrow K^{1} \rightarrow \cdots \rightarrow K^{k} \rightarrow 0
$$

is the Koszul complex of $V$ on $X$.

(b) If, in addition, $X$ is a Banach space, $V_{1}, \ldots, V_{k}$ are continuous and $X_{k}$ is a closed subspace of $X$, then $0 \notin \sigma_{\pi, k-1}(V, X)$.

Proof. Of course (b) follows immediately from (a) and the definition of the spectrum $\sigma_{\pi, k-1}$. Concerning part (a) it is actually formulated, and its proof is indicated in $[16$, p. 119, lines 14-15, Proof of Lemma 1.4]. (The reader should note that Taylor's notation $H_{p}(E(X, V))=0$ is equivalent to the vanishing of the $(k-p)$ th cohomology group of $(4.7)$.)

5. Proof of the main theorem. Theorem 3 is the last step needed to complete the proof of Theorem 1.

PROOF OF THEOREM 3. We can assume, without loss of generality, that $z^{*}=0$, and so $P=\bar{D}\left(0, r_{1}\right) \times \cdots \times \bar{D}\left(0, r_{k}\right), r_{1}>0, \ldots, r_{k}>0$. We take $Y$ as the space $A_{+}(P, X)$ defined in [14] as the space of all $X$-valued analytic functions $f(z)$ defined in the interior of $P$,

$$
f(z)=\sum_{i_{1}, \ldots, i_{k} \geq 0} x_{i_{1}, \ldots, i_{k}} z_{1}^{i_{1}} \cdots z_{k}^{i_{k}}, \quad z \in P
$$

such that

$$
|f|=\sum_{i_{1}, \ldots, i_{k}}\left\|x_{i_{1}, \ldots, i_{k}}\right\| r_{1}^{i_{1}} \cdots r_{k}^{i_{k}}<\infty .
$$

In what follows we will frequently refer the reader to [14] for properties of the space $A_{+}(P, X)$.

Define operators $V_{1}, \ldots, V_{k}$ and $A_{1}, \ldots, A_{m}$ as multiplication operators

$$
\begin{gathered}
\left(V_{i} f\right)(z)=z_{i} f(z), \quad 1 \leq i \leq k, f \in A_{+}(P, X) ; \\
\left(a_{j} f\right)(z)=A_{j}(z) f(z), \quad 1 \leq j \leq m, f \in A_{+}(P, X) .
\end{gathered}
$$

As we observed in [14, Remark 3.1], these formulas define bounded operators of $A_{+}(P, X)$ into itself. It remains to check that the tuple $(V, A)$ satisfies properties (2) and (3). We use the following elementary assertion, whose proof will be given later.

ASSERTION. In the above notations, for every $1 \leq p \leq k$ and for every $\left|a_{1}\right|<$ $r_{1}, \ldots,\left|a_{p}\right|<r_{p}$

$$
\begin{aligned}
& \operatorname{Im}\left(V_{1}-a_{1} I\right)+\cdots+\operatorname{Im}\left(V_{p}-a_{p} I\right) \\
& \quad=\left\{f \in A_{+}(P, X): f(z)=0 \text { whenever } z_{1}=a_{1}, \ldots, z_{p}=a_{p}\right\} .
\end{aligned}
$$


Fix $a$ in the interior of $P$ and denote the subspace just defined by $Y_{p}$. It is easy to observe that the quotient space $Y / Y_{p-1}, 1 \leq p \leq k$, can be identified in a natural way with the space $A_{+}(Q, X)$, where

$$
Q=\left\{\left(a_{1}, \ldots, a_{p}\right)\right\} \times \bar{D}\left(0, r_{p}\right) \times \cdots \times \bar{D}\left(0, r_{k}\right) .
$$

Furthermore, the operator induced by $V_{p}-a_{p} I$ in $Y / Y_{p-1}$ can be identified with the operator of multiplication by $z_{p}-a_{p}$ in $A_{+}(Q, X)$ which obviously has kernel zero. Finally, by (5.1), $Y_{k}$ is a closed subspace of $Y$. Thus the tuple $V_{1}-a_{1} I, \ldots, V_{p}-a_{p} I$ satisfies all the assumptions of Lemma 4.2, and so $a \notin \sigma_{\pi, k-1}(V, Y)$ for $a \in P \backslash \partial P$. On the other hand, by (5.1), $Y_{k} \neq Y$ for $a \in P$, and so $P \subseteq \operatorname{sp}(V, Y)$. Since, by [16, Theorem 3.1], $\operatorname{sp}(V ; Y) \subseteq \bar{D}\left(0,\left\|V_{1}\right\|\right) \times \cdots \times \bar{D}\left(0,\left\|V_{p}\right\|\right)$ and the latter set is equal to $P$. Altogether these observations imply (2).

Concerning (3) we prove first that $\operatorname{sp}(V, A)$ is contained in the set

$$
\{(z, w): z \in P, w \in \operatorname{sp}(T(z), X)\} .
$$

Fix $(a, b) \in \mathbf{C}^{k+m}$ which does not belong to this set. Denote by $d^{0}(z), d^{1}(z), \ldots$, $d^{k+m-1}(z)$ the differentials of the Koszul complex

$$
0 \rightarrow K^{0} \rightarrow K^{1} \rightarrow \cdots \rightarrow K^{k+m-1} \rightarrow K^{k+m} \rightarrow 0
$$

for the tuple $\left(z_{1}-a_{1}\right) I, \ldots,\left(z_{k}-a_{k}\right) I, T_{1}(z)-b_{i} I, \ldots, T_{m}(z)-b_{m} I$. Since, by [16, Lemma 3.1]

$$
\mathrm{sp}(z I, T(z) ; X) \subseteq \mathrm{s}(z I, X) \times \mathrm{sp}(T(z), X)=\{z\} \times \mathrm{sp}(T(z)),
$$

therefore $(a, b) \notin \operatorname{sp}(z I, T(z))$ for $z \in P$ and so complex (5.3) is exact for every $z \in P$ (and so for $z$ in some neigborhood of $P$; cf. e.g. Lemma 1.1). By [14, Corollary 3.5 ] we conclude that the complex

$$
0 \rightarrow A_{+}\left(P, K^{0}\right) \rightarrow A_{+}\left(P, K^{1}\right) \rightarrow \cdots \rightarrow A_{+}\left(P, K^{k+m}\right) \rightarrow 0,
$$

with differentials $\tilde{\delta}^{i}: A_{+}\left(P, K^{i}\right) \rightarrow A_{+}\left(P, K^{i+1}\right), 0 \leq i \leq k+m-1$, defined by

$$
\left(\tilde{\delta}^{i} f\right)(z)=d^{i}(z) f(z), \quad z \in P, f \in A^{+}\left(P, K^{i}\right),
$$

is exact. Now, it is not difficult to check that the natural isomorphisms

$$
A_{+}\left(P, \bigwedge^{i}[e, X]\right) \cong \bigwedge^{i}\left[e, A_{+}(P, X)\right],
$$

where $e=\left(e_{1}, \ldots, e_{k+m}\right)$, identify complex (5.4) with the Koszul complex of the tuple $\left(V_{1}-a_{1} I, \ldots, V_{k}-a_{k} I, A_{1}-b_{1} I, \ldots, A-b I\right)$. Thus $(a, b) \notin \operatorname{sp}(V, A)$, as required.

To complete the proof of (3), fix now arbitrary $a \in P$ and $b \in \operatorname{sp}(T(a), X)$ and consider the smallest $i$ for which the sequence $K^{i} \rightarrow K^{i+1} \rightarrow \cdots \rightarrow K^{k+m} \rightarrow 0$, which is a part of (5.3), is exact for each $z \in P$. By [16, Lemma 3.1] complex (5.3) is not exact at $K^{i}$ for $z=a$; we choose a vector $x_{0} \in \operatorname{ker} d^{i}(a) \backslash \operatorname{Im} d^{i-1}(a)$. Since the relation $\operatorname{Im} d^{i}(z)=\operatorname{ker} d^{i+1}(z)$ holds for all $z$ in some neighborhood of $P$, the function $d^{i}(z)$ satisfies all the equivalent conditions of Theorem 2 in [14], in particular (v). Therefore there exists a function $f \in A_{+}\left(P, K^{i}\right)$ such that $f(a)=x_{0}$ and $d^{i}(z) f(z) \equiv 0$ on $P$. Now it is clear that $f \in \operatorname{ker} \tilde{\delta}^{i} \backslash \operatorname{Im} \tilde{\delta}^{i-1}$ in (5.4), i.e. the Koszul complex of the tuple $(V-a I, A-b I)$ is not exact and so $(a, b) \in \operatorname{sp}(V, A)$, which proves (3). 
It remains to check the assertion. We prove it by induction on $p=1, \ldots, k$. If $p=1$ and $f\left(a_{1}, z_{2}, \ldots, z_{k}\right)=0,\left|z_{i}\right| \leq r_{i}, 2 \leq i \leq k$ and $f \in A_{+}(P, X)$, the function $q_{1}(z)=\left(z_{1}-a_{1}\right)^{-1} f(z)$, as defined on the torus $\partial D\left(0, r_{1}\right) \times \cdots \times \partial D\left(0, r_{k}\right)$, has absolutely convergent Fourier series (note $\left|a_{1}\right|<r_{1}$ ). Since $g_{1}(z)$ is also analytic inside the polydisc, $g_{1} \in A_{+}(P, X)$ and $f=\left(V_{1}-a_{1} I\right) g_{1}$. Assuming now that (5.1) is true for $p$ (with $p<k$ ), we prove it for $p+1$. If $f \in A_{+}(P, X)$ and $f(z)=0$ whenever $z_{1}=a_{1}, \ldots, z_{p+1}=a_{p+1}$, set $f_{1}(z)=f\left(a_{1}, \ldots, a_{p}, z_{p+1}, \ldots, z_{n}\right)$. Of course $f_{1} \in A_{+}(P, X)$ and furthermore, $f_{1}(z)=0$ whenever $z_{p+1}=a_{p+1}$. By case $p=1$ there is $g_{p+1} \in A_{+}(P, X)$ such that $f_{1}=\left(V_{1}-a_{1} I\right) g_{p+1}$. Observe now that $\left(f-f_{1}\right)(z)=0$ whenever $z_{1}=a_{1}, \ldots, z_{p}=a_{p}$. Therefore, by the inductive assumption, $f-f_{1}=\left(V_{1}-a_{1} I\right) g_{1}+\cdots+\left(V_{p}-a_{p} I\right) g_{p}$ for some $g_{1}, \ldots, g_{p} \in A_{+}(P, X)$ and so

$$
f=\left(V_{1}-a_{1} I\right) g_{1}+\cdots+\left(V_{p+1}-a_{p+1} I\right) g_{p+1} \text {. Q.E.D. }
$$

Proof of Theorem 1. Denote $Z=\{(z, w): z \in G, w \in K(z)\}$. Since local maximum property of order $k$ is actually local (cf. e.g. Lemma 1.2) it is enough to check that $K$, restricted to an open polydisc $P$, is an analytic multifunction if $\bar{P} \subset G$. This follows from Theorems 2 and 3 combined. Q.E.D.

6. Concluding remarks. We would like to comment briefly on alternative ways of proving, and possible extensions, of the results of this paper.

A substantial part of the paper ( $\S \S 2$ and 3 ) is occupied by the proof of Theorem 4. Clearly, one can prove it more directly using explicitly the construction of the functional calculus in [16], while our method applies only to some functorial properties of this calculus and does not refer to the construction itself. On the other hand, our treatment of continuous perturbations of the Taylor spectrum and complexes of Banach spaces would have been more natural, if carried over in the general framework of continuous families of Banach spaces, much in the spirit of [13]. While this could be done easily with similar methods, the exposition would be perhaps too long. On the other hand, most of $\S 2$ could be avoided entirely, if one decides to use analytic families of Banach spaces in Theorem 4, and rely on Theorem 5.1 in [14]. This would shorten the proof of Theorem 2, but would make it also appear more difficult.

We note also that Corollary 3.4 can be easily generalized to functions $h(\lambda, z)$, continuous in $(\lambda, z)$ and analytic in $z$.

It seems rather certain that a combination of the methods used in this paper with those of [13 and 14] should yield a generalization of Theorem 1 to the setting of analytic families of Banach spaces as presented in [13].

One can observe that Lemma 4.1 is a immediate consequence of the theory of spectral sequences, cf. [4, §VIII 9].

Finally, the proofs of Theorems 3 and 4 can be considerably simplified in case $X$ is a Hilbert space; in particular there is no need to apply [14].

ACKNowledgment. The author is grateful to Professors E. Effros and T. W. Gamelin for their interest in the results of this work.

ADDED IN PROOF. A partial converse to Theorem 1 has been obtained by the author in [18, Theorem 5.2]. 


\section{REFERENCES}

1. B. Aupetit, Analytic multi-valued functions in Banach algebras and uniform algebras, Adv. in Math. 44 (1982), 18-60.

2. R. F. Basener, Several dimensional properties of the spectrum of a uniform algebra, Pacific J. Math. 74 (1978), 297-306.

3. A. Ya. Khelemskii, Homological methods in Taylor's holomorphic functional calculus of several operators in a Banach space, Russian Math. Surveys 36 (1981), 139-192.

4. P. J. Hilton and U. Stammbach, A course in homological algebra, Graduate Texts in Math., vol. 4, Springer-Verlag, Berlin and New York, 1971.

5. K. Oka, Note sur les familles de fonctions analytiques multiformes, etc., J. Sci. Hiroshima Univ. Ser. A4 (1934), 93-98.

6. T. J. Ransford, Analytic multivalued functions, Dissertation for the Annual Fellowship Competition, Trinity College, Cambridge, 1983.

7. Z. Slodkowski, An infinite family of joint spectra, Studia Math. 61 (1977), 240-255.

8. __ Analytic set-valued functions and spectra, Math. Ann. 256 (1981), 363-386.

9. ___ Analytic families of operators: variation of the spectrum, Proc. Roy. Irish. Acad. Sect. A 81 (1981), 121-126.

10. __, Uniform algebras and multifunctions, Rend. Accad. Naz. Lincei 75 (1983), 9-18.

11. __ Analytic multifunctions, q-plurisubharmonic functions and uniform algebras, Proc. Conf. on Banach Algebras and Several Complex Variables, Yale Univ. (F. Greenleaf and D. Gulick, eds.), Contemp. Math., Vol. 32, Amer. Math. Soc., Providence, R.I., 1983 , pp. 12-14.

12. Local maximum property and q-plurisubharmonic functions in uniform algebras, J. Math. Anal. Appl. (to appear).

13. __ A generalization of Vesentini and Wermer's theorems, Rend. Sem. Mat. Univ. Padova (to appear).

14. __ Operators with closed ranges in spaces of analytic vector-valued functions, J. Funct. Anal. (to appear).

15. Z. Slodkowski and W. Zelazko, On joint spectra of commuting families of operators, Studia Math. 50 (1974), 127-148.

16. J. L. Taylor, A joint spectrum for several commuting operators, J. Funct. Anal. 6 (1970), $172-191$.

17. _ The analytic functional calculus for several commuting operators, Acta Math. 125 (1970), 1-38.

18. Z. Slodkowski, An analytic set-valued selection and its applications to the corona theorem, to polynomial hulls and to joint spectra, Trans. Amer. Math. Soc. 294 (1986), $367-377$.

\section{Department of Mathematics, University of California, Los ANgeles, Cali- FORNIA 90024}

Current address: Department of Mathematics, University of Illinois at Chicago, Chicago, Illinois 60680 